2006 through 2010; clinical data were collected through September 2014. We identified demographic, lifestyle, and medical factors, measured at baseline, that associated with development of EAC within 5 years using logistic regression analysis. We used these data to create a model to identify individuals at risk for EAC. Model performance was assessed using area under the receiver operating characteristics curve (AUROC), sensitivity, and specificity analyses.

Results Within up to 5 years of follow up, 220 individuals developed EAC. Age, sex, smoking, body mass index, and history of esophageal conditions or treatments identified individuals who developed EAC (AUROC, 0.80; 95\% CI, 0.770.82). We used these factors to develop a scoring system and identified a point cut off that 104723 individuals (29.5\%), including 170 of the 220 cases with EAC, were above. The scoring system identified individuals who developed EAC with $77.4 \%$ sensitivity and $70.5 \%$ specificity. The 5 year risk of EAC was $0.16 \%$ for individuals with scores above the threshold and $0.02 \%$ for individuals with scores below the threshold.

Conclusions We combined data on several well-established risk factors that are available to clinicians to develop a system to identify individuals with a higher absolute risk of EAC within 5 years. Studies are needed to evaluate the utility of these factors in a multi-stage, triaged, screening program.

\section{OTU-013 OUTCOMES OF 360 HALO EXPRESS RADIO-FREQUENCY ABLATION FOR BARRETT'S OESOPHAGUS RELATED NEOPLASIA}

\begin{abstract}
${ }^{1,2}$ Cormac Magee*, ${ }^{3}$ Charles Gordon, ${ }^{4}$ Jason Dunn, ${ }^{4}$ Sebastian Zeki, ${ }^{5}$ lan Penman, ${ }^{6}$ Robert Willert, ${ }^{7}$ Howard Smart, ${ }^{8}$ Jacobo Fernandez-Sorto, ${ }^{8}$ Krish Ragunath, ${ }^{8}$ Mirela Pana, ${ }^{9}$ Massimiliano Di Pietro, ${ }^{10}$ Narayanasamy Ravi, ${ }^{11}$ Allan Morris, ${ }^{12}$ Sharmila Subramanian, ${ }^{12}$ Pradeep Bhandari, 'David Graham, ${ }^{1,13}$ Laurence Lovat, ${ }^{1,13}$ Rehan Haidry. ${ }^{1}$ University College London Hospitals, London, UK; ${ }^{2}$ Department of Metabolism and Experimental Therapeutics, University College London, London, UK; ${ }^{3}$ Royal Bournemouth Hospitals, Bournemouth, UK; ${ }^{4}$ Guy's and St Thomas's Hospitals, London, UK; ${ }^{5}$ Royal Infirmary of Edinburgh, Edinburgh, UK; ${ }^{6}$ Central Manchester University Hospitals, Manchester, UK; ${ }^{7}$ Royal Liverpool University Hospitals, Liverpool, UK; ${ }^{8}$ Nottingham University Hospitals, Nottingham, UK; ${ }^{9}$ Hutchison/MRC Research Centre, University of Cambridge, Cambridge, UK; ${ }^{10}$ St James's Hospital, Dublin, UK; ${ }^{11}$ Glasgow Royal Infirmary, Glasgow, UK; ${ }^{12}$ Queen Alexandra Hospital, Portsmouth, UK; ${ }^{13}$ Division of Surgery and Interventional Science, University College London, London, UK
\end{abstract}

\subsection{6/gutjnl-2018-BSGAbstracts.275}

Introduction Radio-frequency ablation (RFA) for the treatment of Barrett's oesophagus (BE) related neoplasia ablative intervention after endoscopic resection (ER)For circumferential BE, the $3 \mathrm{~cm}$ HALO 360 .

balloon used to treat large areas. A new device, the HALO 360 Express self-sizing catheter was recently launched and can potentially allow quicker ablation times and better coverage of the mucosa due to the improved tissue/catheter contact and $4 \mathrm{~cm}$ balloon length. We have previously presented initial data of 3 month follow up in these patients but now present more extensive data including end of treatment biopsies.

Method Specialist centres in the UK and Ireland submitted cases where Halo 360 express had been used. Patients returned for follow up at 3 months after index RFA express treatment. surface area regression of $\mathrm{BE}$ regression of intestinal metaplasia (EoT (End of Treatment) CR-IM) and dysplasia (EoT CR-D) were analysed.[MDP1] .
Results 11 centres submitted 123 patients treated with the HALO 360 Express catheter. 112 of these cases had 3 month follow up. The mean age was 67 years \pm 10 . 83\% were male. 43 patients (35\%) had low grade dysplasia (LGD) as initial histology; 62 had high grade dysplasia (HGD) 50\%, 19 had intramucosal carcinoma (15\%), 1 had invasive adenocarcinoma. 54 (44\%) had had previous endoscopic mucosal resection (EMR). The mean pre-treatment circumferential Barrett's segment was $5.5 \mathrm{~cm} \pm 4.3 \mathrm{~cm}$ and the mean mucosal length (M) was $7.8 \mathrm{~cm} \pm 3.6 \mathrm{~cm}$ mean\% reduction in $\mathrm{C}$ of $78 \% \pm 36 \%$ and mean reduction in $\mathrm{M}$ of $55 \% \pm 36 \%$ at this first 3 month follow up.

17 patients developed strictures which required dilation at this 3 month follow up. The median number of dilations was 2 (IQR2-4). 4/17 (24\%) were treated with 12J/no clean, 10/17 were treated with $10 \mathrm{~J} /$ no clean $(59 \%), 3 / 17(17 \%)$ had been treated with 10J/clean protocol. 8/17 (47\%) had had previous EMR.

47 patients had 12 month EoT biopsies, 40 (85\%) had CRD and $34(76 \%)$ had CR-IM. 4/112 patients $(<4 \%)$ had progressed to invasive cancer at the time of writing. The median number of treatments (focal RFA, EMR, APC (argon plasma coagulation)) to EoT was 2(IQR1-4).

Conclusions The HALO 360 Express catheter shows good reduction in $C$ and $M$ length at 3 months, and effective eradication of IM and Dysplasia in those at 12 months. However, as previously reported by us the stricture rate is high.

\section{OTU-014 REPRODUCTIVE FACTORS AND RISK OF GASTRIC CANCER BY ANATOMICAL SUBSITE: THE EPIC STUDY}

${ }^{1}$ Harinakshi Sanikini*, ${ }^{2}$ Marc Gunter, ${ }^{3}$ Antonio Agudo, ${ }^{3}$ Eric Duell, ${ }^{1}$ Elio Riboli, ${ }^{1}$ Amanda Cross. ${ }^{1}$ Imperial College London, UK; ${ }^{2}$ International Agency for Research on Cancer, France; ${ }^{3}$ Catalan Institute of Oncology, Spain

\subsection{6/gutjnl-2018-BSGAbstracts.276}

Introduction Gastric cancer is more common in men than in women, indicating a potential role for sex hormones in its development. The aetiology of gastric cancer differs by anatomical subsite; however, few studies have compared hormonal and reproductive risk factors by subsite in prospective analyses. We investigated the association between reproductive factors and the risk of gastric cancer by subsite in the European Prospective Investigation into Cancer and Nutrition (EPIC) cohort.

Methods EPIC is an on-going multicentre prospective cohort study, which comprises of 521448 men and women, aged 25-70 years, recruited between 1992-2000 from ten European countries. Questionnaires administered at baseline assessed reproductive factors, including age at menarche, menopause, first pregnancy, and first child birth, as well as parity, breast feeding, menopausal hormonal therapy, and oral contraceptive use. The association between reproductive factors and gastric cancer were examined in Cox proportional hazard models to estimate hazard ratios (HRs) and 95\% confidence intervals (CIs), adjusting for potential confounders.

Results During an average of 14 years of follow up, 83 gastric cardia cancers and 191 gastric non-cardia cancers were diagnosed among 343985 women. Compared to women who had their first pregnancy at an earlier age $(<22$ years $)$, women who had their pregnancy at a later age ( $>26$ years) had a decreased risk of gastric non-cardia cancer (HR 0.55, 95\% CI: 0.32-0.92). In addition, compared with women who had not undergone ovariectomy, women who had a bilateral ovariectomy had an increased risk of gastric non-cardia cancer 